\title{
Exploring the Role of the Tail in Bipedal Hopping through Computational Evolution
}

\author{
Jared M. Moore ${ }^{1}$, Anne K. Gutmann ${ }^{2}$, Craig P. McGowan ${ }^{2}$, and Philip K. McKinley ${ }^{1}$ \\ ${ }^{1}$ Dept. Computer Science and Engineering, Michigan State University, East Lansing, Michigan, USA \\ ${ }^{2}$ Dept. Biological Sciences, University of Idaho, Moscow, Idaho, USA \\ moore112@msu.edu
}

\begin{abstract}
Bipedal hopping has evolved as a mode of terrestrial locomotion in relatively few mammalian species. Despite large differences in body size, habitat use, and having evolved independently, all species that use bipedal hopping have remarkably similar limb morphology and posture. In addition, these species all have relatively long tails, presumably to assist in maintaining stability. However, the evolution of this behavior, and specifically the role of the tail, is not well understood. In this paper, we explore the evolution of bipedal hopping in a simulated animat, using a relatively simple musculoskeletal model and a rigid-body physics simulation environment. Results indicate that characteristically different hopping gaits evolve with alterations to the morphology, including the structure and actuation of the tail. Many of the the results are consistent with behaviors and morphologies observed in natural organisms. However, in some cases effective hopping evolved despite key differences from nature, potentially inspiring new design approaches in robotic and biomechanical systems.
\end{abstract}

\section{Introduction}

Bipedal hopping has evolved in relatively few mammalian species, but apparently for different reasons. In small animals such as kangaroo rats (Figure 1), spring hares, and jerboas, hopping is primarily used as a predator escape mechanism (Biewener and Blickhan, 1988). In larger animals, such as kangaroos and wallabies, hopping offers an energyefficient means of locomotion over long distances (Dawson and Taylor, 1973). Despite size differences, the overall morphologies of these animals are quite similar. Specifically, bipedal hoppers tend to have long tails and powerful hind legs, which perform the majority of work during locomotion.

Yet, the evolutionary origins of this behavior, as well as many related issues, remain obscure. Can bipedal hopping evolve only with this morphology, or is it coincidence that these various species exhibit similar body proportions? Which aspects of the morphology are essential to hopping? Do there exist other morphologies for which bipedal hopping would provide an effective means of locomotion? Not only can answering such questions inform biology, but a better understanding of the evolutionary history and mechanics of hopping has application in biomechanics, robotics and the development of prosthetics. Unfortunately, the relatively small number of species that exhibit this behavior, as well as incomplete fossil records, make it difficult to address this problem through natural systems alone.

Computational evolution provides a means to explore the selective pressures that can lead to hopping, as well as morphological characteristics that sustain it over generations. Moreover, both the behavior and body can deviate from those occurring in nature, enabling the researcher to discover more general principles regarding these issues. A previous study into the evolution of hopping using a $2 \mathrm{D}$ musculoskeletal model found that both quadrupedal and bipedal hopping gaits are very sensitive to changes in morphology (Hase et al., 2004). However, such a model does not take into account many aspects of hopping, such as maintaining balance, that are essential in the physical world. Our work explores the evolution of hopping in 3D physicsbased simulation environments. While our early studies, described here, rely on rigid-body physics environments, more complex musculoskeletal models have been developed (Gutmann et al., 2012) and will be integrated into our investigations as computational capacity permits.

In this paper, we focus on the role of the tail in the evolution of hopping behavior. The virtual animat model approximates muscles, joints, mass and torque, enabling us to evolve biologically plausible patterns of movement. Through a series of five evolutionary treatments, described

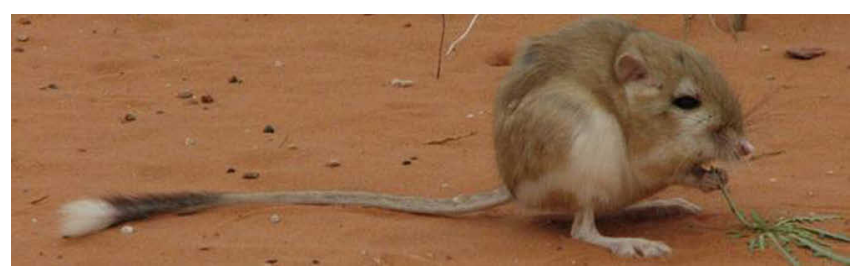

Figure 1: The kangaroo rat was selected as the base morphology for studying the evolution of bipedal hopping, due to its representative morphology and the availability of information on both the mechanics and dynamics of its behavior. 
later, we investigate the effect of different initial (and evolvable) tail configurations on the evolution of effective hopping gaits. We initially start with a fixed morphology resembling a kangaroo rat, but restrictions on the morphology are loosened with each subsequent treatment.

The contributions of this paper are as follows. First, the proposed muscle model produces locomotion patterns similar to those of natural organisms and limits the output potential of each individual joint. This model is computationally less expensive than a musculoskeletal dynamics simulator, enabling the large number of evaluations necessary in evolutionary approaches. Second, the results demonstrate that a tail is essential to hopping, but that different configurations can lead to very different gaits, some closely resembling those of biological counterparts (namely kangaroo rats and wallabies), and others different from any known species. Third, while we observed a close coupling among tail movement and the oscillation frequency of leg joints, we discovered multiple combinations that produced effective bipedal hopping behavior. Finally, we were surprised that many evolved tails had relatively low mass, as it is hypothesized that a heavy tail helps maintain a high moment of inertia in animals, producing a more stable gait. This result might be due to our relatively simple model of the morphology (we plan to use more detailed musculoskeletal models in the future), but might also represent a combination of morphology and behavior that has application outside biology.

\section{Related Work}

The role of the tail in locomotion is of considerable interest within biology. In their studies of geckos, which are not bipedal hoppers, Full and colleagues found that the tail is essential to both orientation control and gait stability (Jusufi et al., 2008; Libby et al., 2012). Alexander and Vernon studied the musculoskeletal system of kangaroos and described the overall mechanical system and the forces exerted during hopping (Alexander and Vernon, 1975). They also first hypothesized that the tail was necessary to balance the angular momentum produced by the swinging legs during hopping. However, to our knowledge no one has yet tested this hypothesis, nor explored its significance in other hopping species.

In robotics, hopping is an intriguing locomotion strategy for its potential energy efficiency and the ability to rapidly change elevation. The latter is particularly important to radio communication, as signal propagation distance is greatly increased by moving transmitters above ground level (Cintrón and Mutka, 2010). Indeed, research in this area has led to the development of small robots capable of both self stabilization and hopping (Zhao et al., 2009). Prior studies on hopping have also addressed mechanics of simple, singlejoint actuated robots that were able to achieve stable hopping gaits (Berkemeier and Fearing, 1998), and single-hop robots have been constructed using pneumatic muscle actu- ators (Niiyama et al., 2007). It has also been shown that combining several hops was more energy efficient than a single, powerful hop, while producing the same jumping height (Aguilar et al., 2012). This efficient hopping motion was discovered after analyzing thousands of results, lending support to harnessing the search capability of evolutionary computation in order to address similar problems.

Evolutionary approaches have been shown to be successful in many robotic and biological applications. Beginning with the foundational work of Brooks and Sims (Brooks, 1992; Sims, 1994), computational evolution has proven effective at producing a diverse range of behaviors. Examples include evolution of neural-based controllers (Cliff et al., 1993; Ijspeert, 2001, 2008) and locomotion strategies for real or simulated robots (Bongard, 2011; Clune et al., 2009; Gomez et al., 2008). Other studies have focused on optimizing morphological components, such as the caudal fin of a robotic fish (Clark et al., 2012) and flexible joints in terrestrial robots (Moore and McKinley, 2012). As noted earlier, the evolutionary computation study conducted by Hase et al. (Hase et al., 2004) found that 2D animats with simulated neuromuscular morphologies were capable of both bipedal and quadrupedal hopping motions similar to their respective biological counterparts. By applying evolutionary approaches to the study of bipedal hopping in 3D animats, we hope to gain insights into this behavior at a level not previously explored.

\section{Methods}

We began our study with an animat based roughly on the morphology of a kangaroo rat, whose gaits have been analyzed extensively with the aid of high-speed, high-resolution video cameras (Gutmann et al., 2013); see Figure 2. We first evolved gaits for fixed morphologies, then allowed evolution of morphological parameters such as limb dimensions, joint output potential and mass distribution.

Virtual Animat. Figure 3 shows the initial animat constructed in the Open Dynamics Engine (Smith, 2012), with body part dimensions corresponding to that of the kangaroo rat. The animat also features a controller that actuates all joints. Kinematic data of the kangaroo rat's hopping gait indicated that the individual joints move in a periodic motion similar to a sine wave. Hence, for this initial study where we focus on steady state hopping gaits, a relatively simple sinusoidal controller was implemented; our ongoing investigations use more complex neural-based controllers. In addition, left/right symmetry was enforced. This decision was made primarily due to the difficulty in evolving a controller for a predefined morphology (unlike nature, where they evolved together). Preliminary experiments found that asymmetric controllers had difficulty achieving stable gaits due to large differences in the length of hind and fore limbs. Moreover, observation of kangaroo rats demonstrates left/right symmetry during hopping. 


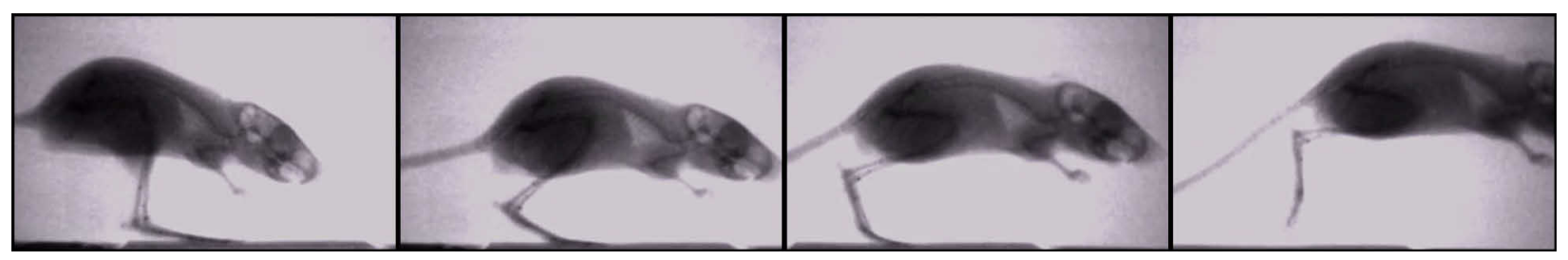

Figure 2: X-ray video progression of a kangaroo rat hopping across a force plate to quantify hopping dynamics.

Muscle Model. Animals exhibit fluid movements produced by muscles contracting and relaxing in a coordinated manner. To approximate such dynamics in a rigid-body simulator such as ODE, we modeled muscular connections using hinge joints with appropriate constraints. In particular, we devised a model in which the energy an individual joint can expend during actuation is limited. Doing so prevents situations in which a joint can move with an infinite amount of force, an impossibility in biological organisms. Figure 4 shows the range of motion and relative power of each joint in the morphology. Limiting the maximum force an individual joint can exert produces a system in which multiple joints must work together to move the animat. This muscular model is applied only to the rear legs, as the fore legs do not factor heavily into the locomotion pattern for evolved individuals.

We found that this model produced coordination among the components of the body and natural looking gaits. During locomotion, animal joints do not always move throughout their entire range of motion (for example, strides may be shortened to handle rough terrain, or the center of gravity may be lowered by crouching to improve balance). If the potential were unlimited, joints would always move throughout their full range of motion, irrespective of external forces. By limiting potential, the range of motion of one joint would be indirectly determined by the evolved muscle output parameters of other joints. Moreover, limiting the overall output potential of each joint allowed the limbs to flex and react to the ground when landing, increasing stability and the "naturalness" of the gait.

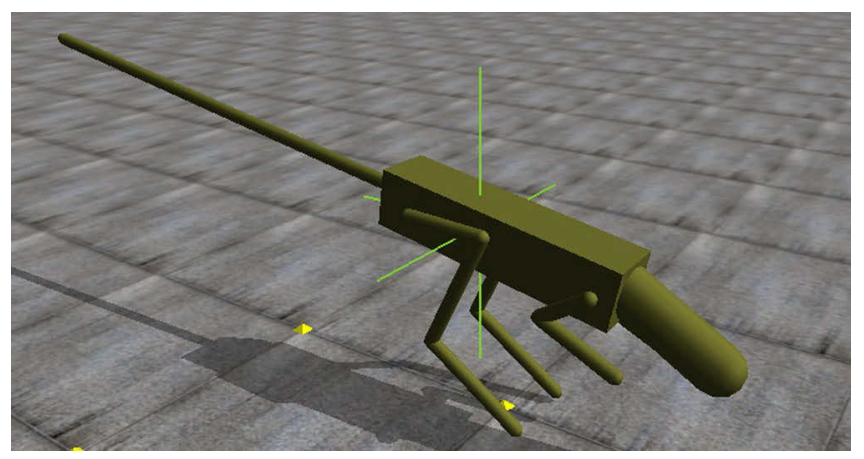

Figure 3: Initial simulated animat used in this study, with morphological dimensions and mass based on kangaroo rat.

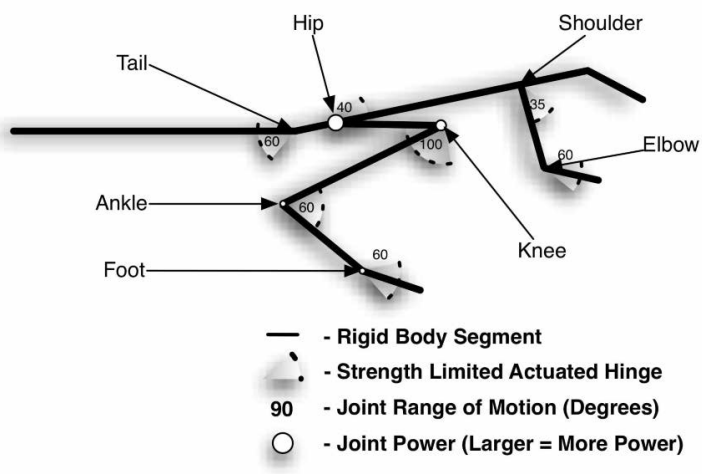

Figure 4: Two-dimensional representation of the animat joints, with range of movement indicated.

Evolutionary Setup. For each of five treatments, described in the next section, we executed 25 replicate runs, each with a unique random number seed. In each run, a population of 150 individuals evolved for 4000 generations. Fitness was defined simply to be the distance traveled in 10 seconds of simulated time. No special selective pressure was applied to prefer hopping to other forms of locomotion. Successive generations were populated using 2-way tournament selection with mutation and crossover as defined below. The genome comprised 12,14, or 16 values, depending on the treatment, as shown in Table 1. For treatments 1 and 2, the genome did not include parameters for an actuated tail.

The mutation rate was relatively high, $20 \%$, but mutations were defined according to a gaussian distribution, so an individual mutation was unlikely to produce a large change in value. We found this approach to be effective given the control strategy used, where a large change in a single key parameter, such as a phase offset, often produced an unstable solution. A more conservative mutation approach allowed for gradual change to gait patterns over generations.

Single-point crossover was applied with a probability of $25 \%$ per genome. Crossover exhibited spatial locality, in that parents for an individual solution were chosen within a defined range. Specifically, we applied a geographical approach (Spector and Klein, 2006), where the population is considered as a one-dimensional line with wrap-around. Individuals are produced from parents that are considered to be close to their offspring. 
Table 1: Individual Gene Limits

\begin{tabular}{|c|l|l|}
\hline Parameter & Min. Value & Max Value \\
\hline Actuation Freq. & $0 \mathrm{~Hz}$ & $2.5 \mathrm{~Hz}$ \\
Hip Orientation & $0^{\circ}$ & $337.5^{\circ}$ \\
Knee Orientation & $0^{\circ}$ & $337.5^{\circ}$ \\
Ankle Orientation & $0^{\circ}$ & $337.5^{\circ}$ \\
Toe Orientation & $0^{\circ}$ & $337.5^{\circ}$ \\
Shoulder Orientation & $0^{\circ}$ & $337.5^{\circ}$ \\
Elbow Orientation & $0^{\circ}$ & $337.5^{\circ}$ \\
Center of Mass & body center - & body center + \\
& $0.25 \times$ length & $0.25 \times$ length \\
Hip Power & 0 (passive) & 1.0 \\
Knee Power & 0 (passive) & 1.0 \\
Ankle Power & 0 (passive) & 1.0 \\
Toe Power & 0 (passive) & 1.0 \\
\hline \multicolumn{2}{|c|}{ Treatments 3,4 and 5 } \\
\hline Tail Actuation Freq. & $0 \mathrm{~Hz}$ & $2.5 \mathrm{~Hz}$ \\
Tail Orientation & $0^{\circ}$ & $337.5^{\circ}$ \\
\hline \multicolumn{2}{|c|}{ Treatment 5 Only } \\
\hline Tail Length & $0.07 \times$ body & $2.2 \times$ body \\
Tail Mass & length & length \\
\hline \multicolumn{2}{|c|}{ body mass } & $0.6 \times$ body \\
mass
\end{tabular}

\section{Experiments \& Results}

The 5 treatments, described below, investigate the role of the tail in bipedal hopping, including interaction with other aspects of the morphology and effect on gaits. To assist the reader in visualizing evolved behaviors, we have placed videos of selected evolved behaviors on a YouTube account:

Treatment 1: http://y2u.be/V56Xmgf 7pxE Treatment 2: http://y2u.be/M1BWXwVUAEM Treatment 3: http://y2u.be/bizIMorOv9g Treatment 4: http://y2u.be/dIyoE0eMm2A Treatment 5: http://y2u.be/OXIbXrwXU3Y

Treatment 1: No Tail. In Treatment 1, individuals lack a tail. Most (18) of the 25 replicate runs failed to produce bipedal hopping, instead evolving bounding gaits, where fore and hind limbs alternate contact with the ground. Such gaits were common throughout the study, since they offer relatively stable locomotion, albeit slower than bipedal hopping. Six of the replicate runs were able to manage two or three hops before settling into a forward-leaning gait and then regressing to a bounding gait. However, in one run, the dominant individual, shown in Figure 5 and the Treatment 1 video, exhibited a fairly effective bipedal hopping gait, although it flipped over near the end of the simulation period. Presumably, the bounding gait was a more stable configuration for tailless animats. Examination of early generations found that many individuals attempting to hop tended to flip over backwards, resulting in low fitness scores. One encouraging trend that emerged in this and subsequent treatments was the effectiveness of our muscle model in simulating flexible joints. During locomotion, joints flexed to react to contact with the ground, resembling the function of biological musculoskeletal systems.

Treatment 2: Fixed, Rigid Tail. In the second treatment, individuals had a fixed, rigid tail, and were able to evolve hopping gaits with relatively high fitness values. However, we observed that the majority of successful hoppers used the tail as a "kickstand" to prevent flipping over, as had occurred in Treatment 1 . The increased stability enabled individuals to hop farther. The best evolved individual for this treatment can be seen in Figure 5. Most of the replicate runs produced individuals that used their tail in this manner through the entire simulation period, however, a few managed to execute two or three hops between tail taps. Although not ideal, this tail-tapping motion turned out to be an important aspect in the emergence of hopping gaits.

Treatment 3: Actuated Tail. The fixed tail in Treatment 2 approximates the initial posture of a kangaroo rat at the start of a hopping motion. In Treatment 3 , we expanded the genome to allow the tail to evolve a speed of oscillation value as well as a starting position. We expected to see hopping gaits that did not use the tail as a kickstand as had occurred in Treatment 2. Evolved solutions for this treatment did tend to favor oscillating tails that counteracted the angular momentum of the body. However, the kickstand effect was still present in many individuals, although not as predominant as those evolved previously. In addition to the kickstand function of the tail, evolved individuals demonstrated a coupling between tail and leg oscillation that has the tail moving against the legs to limit the rotation of the body during the hop. An evolved individual for this treatment can be seen in Figure 6, which shows the use of the actuated tail to stabilize the body pitch.

Treatment 4: Tail Collision Removal. In a natural environment, hopping species tend not to drag their tails on the ground or even allow the tail to contact the ground at high speeds, in order to avoid injury. In Treatment 4, we explicitly removed the kickstand effect by simply preventing the tail from interacting with the ground. (Effectively, the tail could contact and penetrate the ground with no effect on the animat.) We expected solutions to instead use the tail as a counterbalance to angular momentum, consistent with a prevailing hypothesis in biology (Bartholomew and Caswell, 1951; Alexander and Vernon, 1975; Libby et al., 2012). Instead, the results from all replicate runs tended towards bounding gaits similar to those in Treatment 1 . We suspect that the additional mass associated with a tail made it more difficult for the individuals to maintain balance, resulting in the tendency to lean forward. 


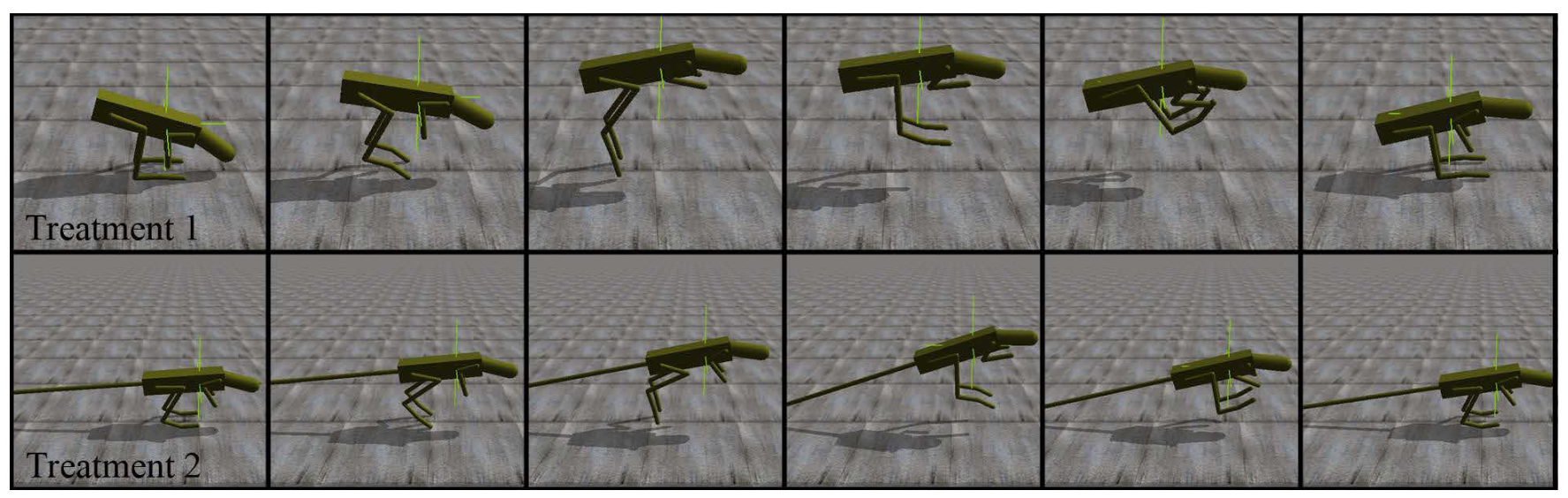

Figure 5: Behavior of evolved tailless and fixed-tail individuals. The fixed tail individual was able to hop more effectively by using its tail as a stabilizer to prevent flipping over backwards.

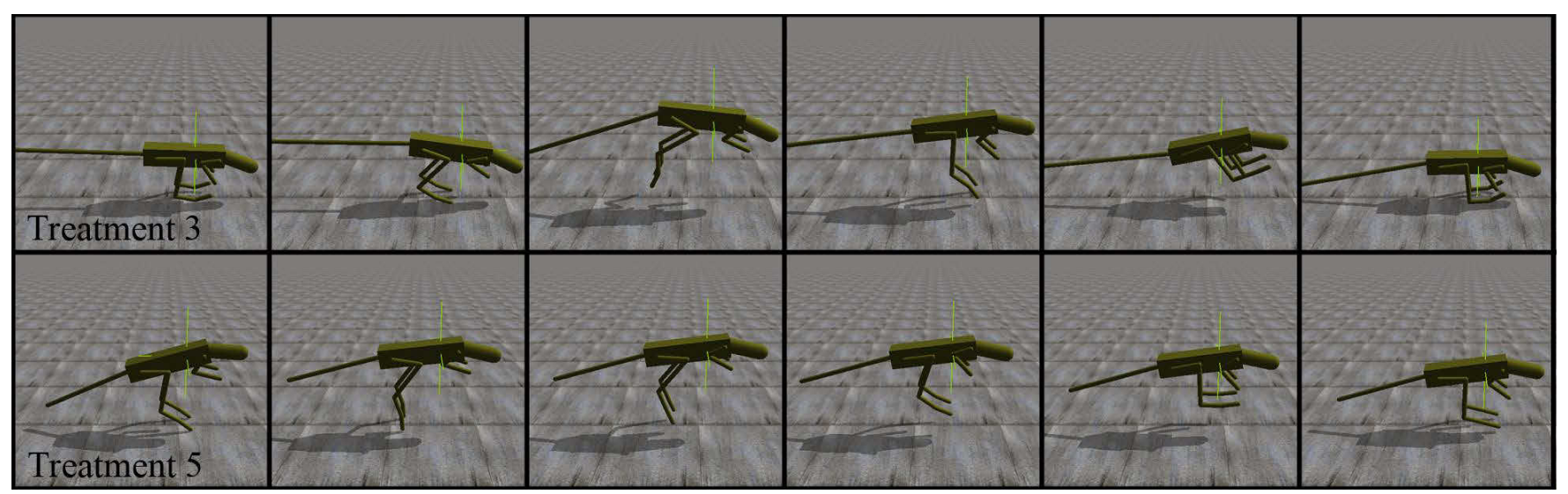

Figure 6: An evolved hopping individual from Treatment 3 with an actuated tail. Note the coordination between tail and legs to maintain body pitch throughout the hopping motion. In the evolved individual from Treatment 5 , the tail evolves to be shorter than those of the previous treatments, enabling faster hopping.

Treatment 5: Evolvable Tail Morphology In the first four treatments, tails appeared to be essential to maintaining stability. In biology, it is generally agreed that an important function of the tail is to counter the angular momentum of the body, discouraging body pitch changes over the hopping period (Bartholomew and Caswell, 1951; Libby et al., 2012). Since we had based the animat's morphology on the kangaroo rat, we were curious what solutions would be discovered if tail length and tail mass were allowed to evolve. Indeed, Treatment 5 runs produced bipedal hoppers with tails approximately half as long as those in the earlier treatments; an example is shown in Figure 6.

Performance Comparison. Figure 7 plots the best and average fitness for each of the 5 treatments. In Treatment 1 , solutions were forced to focus heavily on stable locomotion rather than maximizing the speed of movement, resulting in low fitness. Treatment 4 exhibited even worse performance in both plots, demonstrating that in these experiments tail tapping is an important part of the behavior, at least as the animat starts moving. Treatment 5 had the best performing individuals across all treatments, although the average performance was similar to that of Treatment 3 . This result is likely due to individuals that were unstable and attained low fitness scores. Individuals in Treatment 2 had the second best performance, presumably by using the tail to stabilize the animat during hopping. Treatment 2 also had the best average fitness, indicating that the static nature of the morphology likely made finding stable solutions easier.

Analysis. Considering the high performance achieved in Treatment 5, we sought to determine which factors and relationships gave rise to effective bipedal hopping. We discovered that in the top $10 \%$ of evolved solutions in this treatment, there existed a relatively tight coupling between tail and leg oscillation frequencies. Figure 8 presents these data 


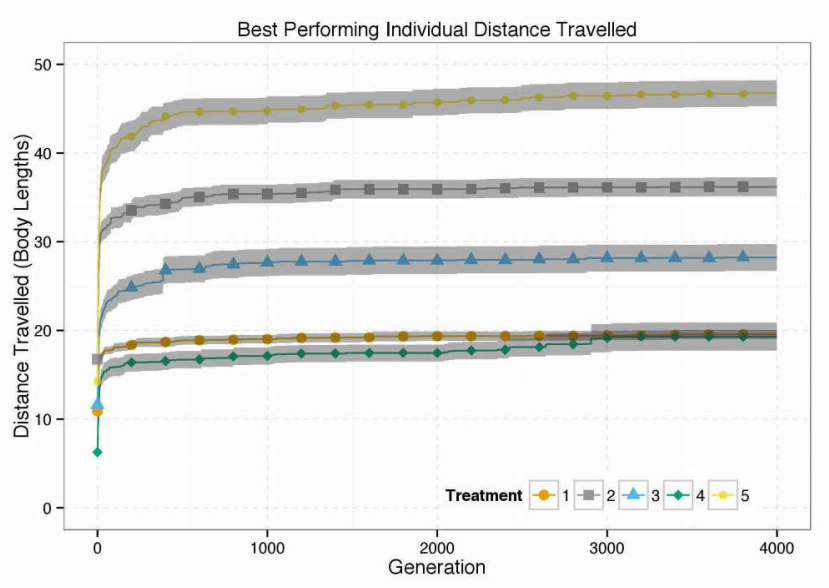

(a)

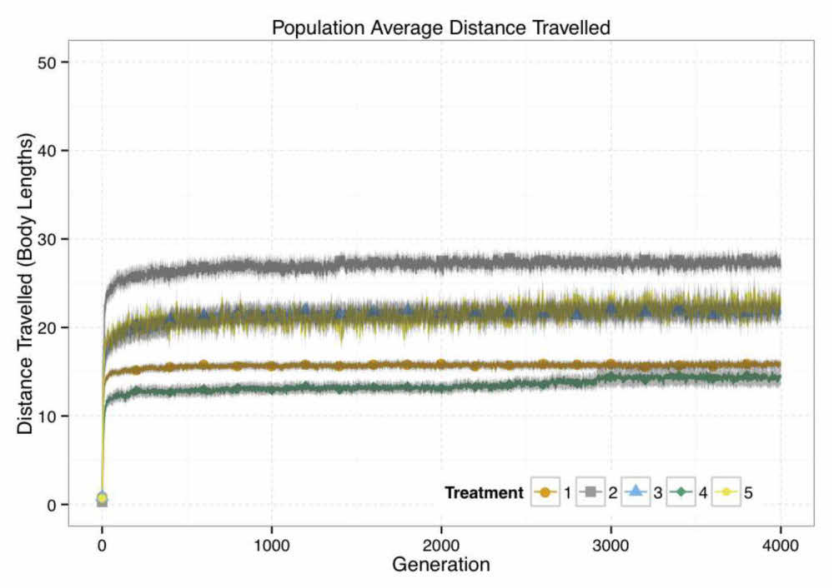

(b)

Figure 7: Fitness of 5 treatments over evolutionary time: (a) Best performing individual, averaged across 25 runs for each treatment; (b) Average performance in each evolved population, averaged across 25 runs for each treatment. Shaded bands indicate $95 \%$ confidence intervals.

for individuals in the final generation. In the figure, the tail oscillation frequencies are generally near either a harmonic of the leg oscillation frequency, or they act as a passively flexible joint (lower right). Results that fall on or near these harmonic values have tails that move directly opposite to the rotation of the body, apparently helping to maintain a more effective body orientation. In the solutions indicated as passively flexible, the tail oscillation frequencies are so low that they behave as a flexible joint that moves only in reaction to the hopping motion, thus countering rotational movement. The coordination in phase between tail and leg movement is essential for successful individuals and is supported by biological observation. In hopping species, tails tend to move in concert with the rest of the body producing a unified gait pattern. In our observations of evolved animats, individu- als lacking this coordination tend to produce extraneous or detracting movements that actually hinder performance.

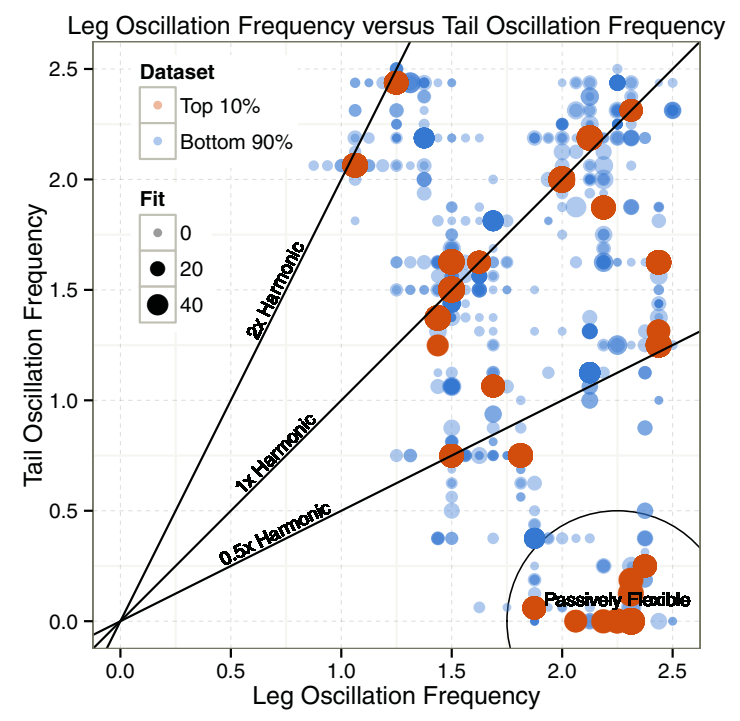

Figure 8: Relationship between the leg oscillation frequency and tail oscillation frequency in Treatment 5. The straight lines indicate harmonics between the two frequencies. Evolved solutions tended to either fall near these lines or in the passively flexible region.

A second area of interest is the evolved mass of the tails and the resulting moments of inertia. As seen in Figure 9, the evolved results tended towards tail masses that were less than $15 \%$ of the total body mass. Indeed, tails in some of the best performing individuals accounted for less than 5\% of total body mass. These light tails resulted in relatively low moments of inertia, as seen in Figure 10. Lower moments of inertia in these individuals potentially allow the body to generally change pitch throughout the hopping motion rather than maintain a stable body orientation.

This result is intriguing because stable orientation in hopping species benefits from a high moment of inertia in tails (Usherwood and Hubel, 2012). Moreover, Figure 10 indicates that there is no direct relationship between the tail moment of inertia and leg oscillation frequency. A possible explanation is related to our evaluation period. While the insight into high moments of inertia for the tails is well understood, the biological observations leading to this conclusion generally focus on steady-state hopping. However, in our treatments, fitness evaluation begins at the start of the simulation period which includes the startup phase. Hence, individuals begin from a stationary starting position and must begin to hop before reaching their final steady state. The inclusion of the startup period places an emphasis on stability during the transition from stationary pose to hopping to avoid falling over or becoming unstable. This pressure 


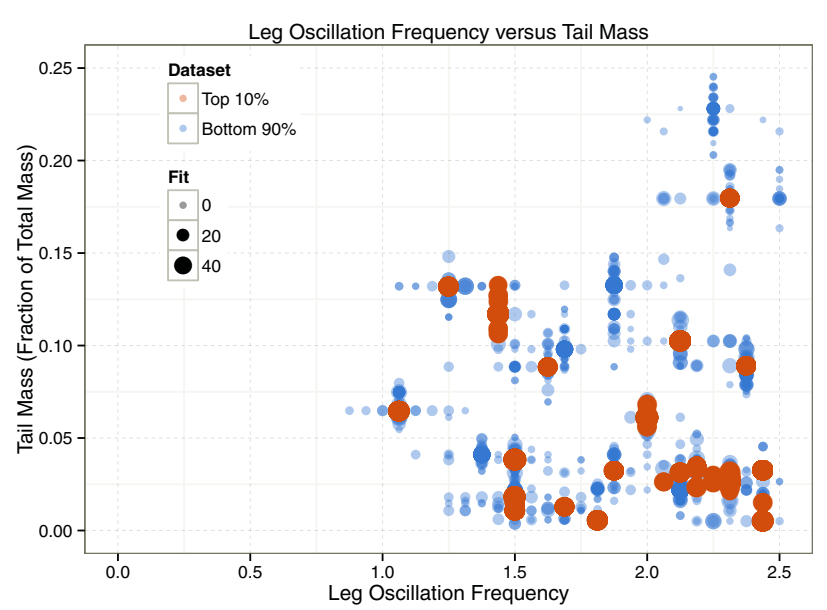

Figure 9: Relationship between the leg oscillation frequency and tail mass as a percentage of total body mass. Lighter tails are favored, although the evolved tail length remains relatively constant even for different masses.

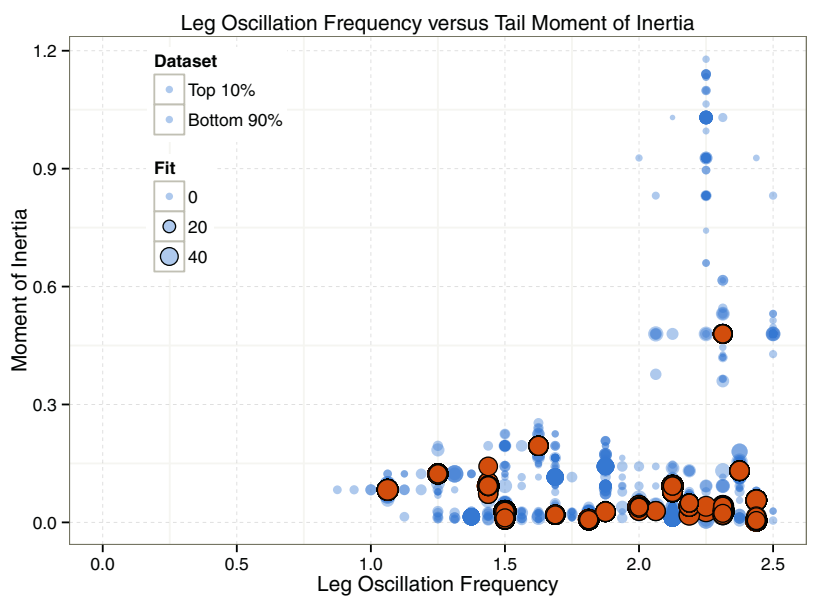

Figure 10: Relationship between the leg oscillation frequency and moment of inertia for an individual. A low moment of inertia generally means the animat is likely to change body pitch during hopping.

likely forces the solutions to evolve parameters that encourage stable startup gaits over those that are most efficient or fastest during the steady-state phase. One possible approach is to delay the evaluation until the animat has had an opportunity to start moving. Adding such a transient phase, which has proven successful in other recent studies (Moore et al., 2013), may encourage tail parameter evolution towards steady state hopping. However, we note that at the time of this writing, a preliminary set of experiments showed that a transient phase actually reduced fitness. This issue is a topic of our ongoing research.

\section{Conclusions}

Although relatively uncommon in the animal kingdom, bipedal hopping provides benefits both for energy efficiency and as a survival mechanism. A better understanding of this behavior, and how it evolved, not only informs biology but has implications for the design of robotic systems. We have developed a computationally-efficient kinematic model that approximates the function of natural muscles and is suitable for integration into evolutionary algorithms. In 5 treatments, we explored the role of the tail in hopping gaits. We found that a tail is essential to hopping, as tailless individuals resorted to bounding or shuffling gaits. Evolved gaits exhibit similarities to their biological counterparts in terms of tail movement and joint coordination. However, our results also show that bipedal hopping is not limited to the morphological configurations observed in nature, but can evolve in other morphologies (i.e., those with short, light tails). Indeed, the initial morphology based on the kangaroo rat dimensions proved not to be the most effective morphology. Finally, the inclusion of the startup phase in fitness evaluation led to an alternate use for the tail as a stabilizer, which to our knowledge has not been previously reported.

In future work we plan to conduct more in-depth study of transient versus steady-state hopping behaviors and the pressures influencing them. We also plan to refine our simulated muscular model to more accurately capture the behavior of natural muscle and tendon systems. Finally, as with our studies of aquatic robots, we intend to evolve more complex controllers, using artificial neural networks, for bipedal hoppers.

\section{Acknowledgments}

The authors gratefully acknowledge the BEACON Center for the Study of Evolution in Action, which directly supported this study and fosters an environment for interdisciplinary collaboration. This research also has been supported in part by U.S. National Science Foundation grants CNS-1059373, CNS-0915855, CNS-0751155, CCF0820220, and DBI-0939454.

\section{References}

Aguilar, J., Lesov, A., Wiesenfeld, K., and Goldman, D. (2012). Lift-off dynamics in a simple jumping robot. Physical Review Letters, 109(174301).

Alexander, R. M. and Vernon, A. (1975). The mechanics of hopping by kangaroos (macropodidae). Journal of Zoology, 177(2):265-303.

Bartholomew, G. A. and Caswell, H. H. (1951). Locomotion in kangaroo rats and its adaptive significance. $J$. Mamm., 32:155-169.

Berkemeier, M. and Fearing, R. (1998). Sliding and hopping gaits for the underactuated acrobot. IEEE Transactions on Robotics and Automation, 14(4):629 -634. 
Biewener, A. A. and Blickhan, R. (1988). Kangaroo rat locomotion: design for elastic energy storage or acceleration? J. Exp. Biol., 140:243-255.

Bongard, J. C. (2011). Morphological and environmental scaffolding synergize when evolving robot controllers. In Proceedings of the 2011 ACM Genetic and Evolutionary Computation Conference.

Brooks, R. A. (1992). Artificial life and real robots. In Proceedings of the First European Conference on Artificial Life, pages 3-10. MIT Press, Cambridge, MA.

Cintrón, F. and Mutka, M. (2010). Hopping enhanced sensors for efficient sensor network connectivity and coverage. In 2010 IEEE 7th International Conference on Mobile Adhoc and Sensor Systems (MASS), pages 119 -126, San Francisco, CA, USA. IEEE.

Clark, A. J., Moore, J. M., Wang, J., Tan, X., and McKinley, P. K. (2012). Evolutionary design and experimental validation of a flexible caudal fin for robotic fish. In Proceedings of the 13th International Conference on the Simulation and Synthesis of Living Systems, East Lansing, Michigan, USA.

Cliff, D., Husbands, P., and Harvey, I. (1993). Explorations in Evolutionary Robotics. Adaptive Behavior, 2(1):73110.

Clune, J., Beckmann, B. E., Ofria, C., and Pennock, R. T. (2009). Evolving coordinated quadruped gaits with the HyperNEAT generative encoding. In Proceedings of the IEEE Congress on Evolutionary Computing, pages 2764-2771, Trondheim, Norway.

Dawson, T. J. and Taylor, C. R. (1973). Energetic cost of locomotion in kangaroos. Nature, 246(5431):313-314.

Gomez, F., Schmidhuber, J., and Miikkulainen, R. (2008). Accelerated neural evolution through cooperatively coevolved synapses. Journal of Machine Learning Research, 9:937-965.

Gutmann, A. K., Lee, D. V., and McGowan, C. P. (2013). Collision dynamics of bipedal hopping. In Annual Meeting of the Society for Integrative and Comparative Biology, San Francisco, California, USA.

Gutmann, A. K., McKinley, P. K., and McGowan, C. P. (2012). A detailed musculoskeletal model for studying the evolution of bipedal hopping. Bio/computational Evolution in Action Consortium Congress, East Lansing, Michigan, USA.

Hase, K., Khang, G., and Eom, G.-M. (2004). A simulation study on the evolution of hopping motions in animals. IEEE Transactions on Systems, Man, and Cybernetics, Part C: Applications and Reviews, 34(3):353 -362.

Ijspeert, A. J. (2001). A connectionist central pattern generator for the aquatic and terrestrial gaits of a simulated salamander. Biological Cybernetics, 84(5):331-348.
Ijspeert, A. J. (2008). Central pattern generators for locomotion control in animals and robots: A review. Neural Networks, 21(4):642-653.

Jusufi, A., Goldman, D. I., Revzen, S., and Full, R. J. (2008). Active tails enhance arboreal acrobatics in geckos. Proceedings of the National Academy of Sciences, 105(11):4215-4219.

Libby, T., Moore, T. Y., Chang-Siu, E., Li, D., Cohen, D. J., Jusufi, A., and Full, R. J. (2012). Tail-assisted pitch control in lizards, robots and dinosaurs. Nature, 481(7380):181-184.

Moore, J. M., Clark, A. J., and McKinley, P. K. (2013). Evolution of station keeping as a response to flows in an aquatic robot. In Proceedings of the 2013 ACM Genetic and Evolutionary Computing Conference, Amsterdam, Netherlands. ACM.

Moore, J. M. and McKinley, P. K. (2012). Evolving flexible joint morphologies. In Proceedings of the 2012 ACM Genetic and Evolutionary Computing Conference, Philadelphia, Pennsylvania, USA. ACM.

Niiyama, R., Nagakubo, A., and Kuniyoshi, Y. (2007). Mowgli: A bipedal jumping and landing robot with an artificial musculoskeletal system. In Proceedings of the 2007 IEEE International Conference on Robotics and Automation, pages 2546 -2551, Roma, Italy.

Sims, K. (1994). Evolving virtual creatures. In Proceedings of the 21st Annual Conference on Computer Graphics and Interactive Techniques, pages 15-22.

Smith, R. (2012). Open Dynamics Engine. Manual and source code available online at: http://www.ode.org.

Spector, L. and Klein, J. (2006). Trivial geography in genetic programming. In Yu, T., Riolo, R., and Worzel, B., editors, Genetic Programming Theory and Practice III, volume 9 of Genetic Programming, pages 109-123. Springer US.

Usherwood, J. R. and Hubel, T. Y. (2012). Energetically optimal running requires torques about the centre of mass. Journal of The Royal Society Interface, 9(73):20112015.

Zhao, J., Yang, R., Xi, N., Gao, B., Fan, X., Mutka, M. W., and Xiao, L. (2009). Development of a miniature self-stabilization jumping robot. In Proceedings of the 2009 IEEE/RSJ international conference on Intelligent robots and systems, pages 2217-2222, St. Louis, MO, USA. IEEE Press. 